University of Texas at El Paso

ScholarWorks@UTEP

$10-2014$

\title{
Why Ricker Wavelets Are Successful in Processing Seismic Data: Towards a Theoretical Explanation
}

\author{
Afshin Gholamy \\ The University of Texas at El Paso, afshingholamy@gmail.com \\ Vladik Kreinovich \\ The University of Texas at El Paso, vladik@utep.edu
}

Follow this and additional works at: https://scholarworks.utep.edu/cs_techrep

Part of the Computer Engineering Commons

Comments:

Technical Report: UTEP-CS-14-47b

Published in Proceedings of the IEEE Symposium on Computational Intelligence for Engineering Solutions CIES'2014, Orlando, Florida, December 9-12, 2014, pp. 11-16.

\section{Recommended Citation}

Gholamy, Afshin and Kreinovich, Vladik, "Why Ricker Wavelets Are Successful in Processing Seismic Data: Towards a Theoretical Explanation" (2014). Departmental Technical Reports (CS). 861.

https://scholarworks.utep.edu/cs_techrep/861

This Article is brought to you for free and open access by the Computer Science at ScholarWorks@UTEP. It has been accepted for inclusion in Departmental Technical Reports (CS) by an authorized administrator of ScholarWorks@UTEP. For more information, please contact Iweber@utep.edu. 


\title{
Why Ricker Wavelets Are Successful in Processing Seismic Data: Towards a Theoretical Explanation
}

\author{
Afshin Gholamy and Vladik Kreinovich \\ Computational Science Program \\ University of Texas at El Paso \\ $500 \mathrm{~W}$. University \\ El Paso, Texas 79968, USA \\ afshingholamy@gmail.com,vladik@utep.edu
}

\begin{abstract}
In many engineering applications ranging from engineering seismology to petroleum engineering and civil engineering, it is important to process seismic data. In processing seismic data, it turns out to be very efficient to describe the signal's spectrum as a linear combination of Ricker wavelet spectra. In this paper, we provide a possible theoretical explanation for this empirical efficiency. Specifically, signal propagation through several layers is discussed, and it is shown that the Ricker wavelet is the simplest non-trivial solution for the corresponding data processing problem, under the condition that the described properties of the approximation family are satisfied.
\end{abstract}

\section{Formulation of the Problem: Seismic WaVes AND THE EMPIRICAL SUCCESS OF RICKER WAVELETS}

Seismic data is very useful. Already ancient scientists noticed that earthquakes generate waves which can be detected at large distances from the their origin. These waves were called seismic waves, after the Greek word "seismos" meaning an earthquake.

After a while, scientists realized that from the seismic waves, we can extract not only important information about earthquakes, but also information about the media through which these waves propagate. Different layers reflect, refract, and/or delay signals differently, so by observing the coming waves, we can extract a lot of information about these layers. Since earthquakes are rare, geophysicists set up small artificial explosions or vibrations that also serve as a source of seismic waves.

The resulting seismic information helps geophysicists, petroleum and mining engineers, hydrologists to find mineral deposits and underground water reservoirs, helps civil engineers get a good understanding of the stability of the underground layers below the future structure (e.g., a building, a tunnel, a dam, or a landfill) [1], [5], [19], etc.; see, e.g., [14].

In particular, computational intelligence techniques are actively used in processing seismic data; see, e.g., [6] and references therein.

Ricker wavelet: reminder. To describe how the amplitude $x(t)$ of a seismic signal changes with time $t, \mathrm{~N}$. Ricker proposed, in his 1953 paper [16], to use a linear combination of wavelets of the type

$$
x(t)=\left(1-\frac{\left(t-t_{0}\right)^{2}}{\sigma^{2}}\right) \cdot \exp \left(-\frac{\left(t-t_{0}\right)^{2}}{2 \sigma^{2}}\right)
$$

corresponding to different moments of time $t_{0}$ and different values of the parameter $\sigma$ describing the duration of this wavelet signal.

The power spectrum $S(\omega)$ of this wavelet has the form

$$
S(\omega)=K \cdot \omega^{2} \cdot \exp \left(-c \cdot \omega^{2}\right),
$$

where $c=\sigma^{2}$ and $K$ is a constant.

Ricker wavelets have been empirically successful. Since the original Ricker's paper, Ricker wavelets have been successfully used in processing seismic signal; see, e.g., [2], [3], [4], [5], [8], [9], [10], [12], [13], [18], [20] (see especially [4]). Ricker wavelet are also actively used when computational intelligence techniques are used for processing seismic data; see, e.g., [6].

Specifically, in line with Ricker's suggestion, the power spectrum $S(\omega)$ of the seismic signal is represented as a linear combination of power spectra corresponding to Ricker wavelets:

$$
S(\omega) \approx \sum_{i=1}^{n} K_{i} \cdot \omega^{2} \cdot \exp \left(-c_{i} \cdot \omega^{2}\right) .
$$

To describe each spectrum term $K_{i} \cdot \omega^{2} \cdot \exp \left(-c_{i} \cdot \omega^{2}\right)$, we need to use two parameters: $K_{i}$ and $c_{i}$. Thus, overall, we need $2 n$ parameters in the above approximation (1), where $n$ is the number of terms used in this approximation.

This Ricker wavelet approximation is often empirically successful in the sense that for the same approximation accuracy, it usually requires fewer parameters than other possible approximations; see, e.g., [4], [9].

Need for a theoretical explanation. Empirical studies have shown that Ricker wavelets, in general, lead to a better approximation of the seismic spectra than other families of approximating functions. However, in principle, there are many possible families of approximating functions, and only few of these families were actually tested.

So, a natural question arises: are Ricker wavelets indeed the best or are they just a good approximation to some even better (not yet known) family of approximating functions?

To answer this question, we will perform a theoretical analysis of the problem, and we will show that Ricker wavelets are the best. Specifically, our analysis of the problem of approximating seismic spectra will lead us to several reasonable 
properties that the approximating family must satisfy; then, we will show that Ricker wavelets are the only approximating family which satisfies these properties.

\section{ANALYSIS OF THE PROBLEM}

How each propagation layer affects the seismic signal. Layers are not homogeneous. As a result, the same seismic signal, when passing through different locations on the same layer, can experience different time delays. Hence, even when we start with a pulse signal, a signal which is different from 0 only at one moment of time $t$, this pulse comes to the next point via several possible trajectories on each of which the time delay is slightly different. Thus, a unit pulse signal at moment 0 is transformed into a signal $m(t)$ which is distributed in time.

Seismic signals are weak. For weak signals, we can expand the dependence of the output on the input in Taylor series and keep only linear terms in this expansion. Thus, the output signal linearly depends on the input one. Hence, if a unit pulse gets transformed into a signal $m(t)$, a pulse of size $x$ get transformed into a signal $m(t) \cdot x$.

In seismic measurements, the media does not change with time. So, if we shift the original pulse signal $s$ seconds in time, making it a unit pulse at size $x$ at moment $s$, the transformed signal will also be equal to the similar shift of the signal

$$
m(t) \cdot x,
$$

i.e., to the signal $m(t-s) \cdot x$.

In the discrete-time approximation, with a time step $\Delta t$, an arbitrary signal $x(t)$ can be represented as a sum of pulses of size $x(s)$ occurring at different moments of time $s$. Each such pulse leads to the transformed signal $m(t-s) \cdot x(s)$. Thus, due to linearity, the sum of the pulses gets transformed into the sum of such transformed signals, i.e., into $\sum m(t-s) \cdot x(s)$, when the sum is taken over the grid with width $\Delta t$. In the limit when $\Delta t \rightarrow 0$, we get a more and accurate representation of the original signal $x(t)$ and thus, a more and more accurate representation of the transformed signal.

One can easily see that the sum $\sum m(t-s) \cdot x(s)$ is an integral sum for an integral $\int m(t-s) \cdot x(s) d s$, and the limit $\Delta t \rightarrow 0$, the sum does tends to the integral. Thus, each layer transforms the original signal $x(t)$ into the new signal

$$
\int m(t-s) \cdot x(s) d s
$$

for details, see, e.g., [7], [14].

What is the joint effect of propagating the signal through several layers? Let us assume that we started with the signal $x_{0}(t)$. This signal passes through the first layer, and is thus transformed into

$$
x_{1}(t)=\int m_{1}(t-s) \cdot x_{0}(s) d s .
$$

The resulting signal $x_{1}(t)$ then passes through the second layer, and is, thus, transformed into

$$
y(t)=\int m_{2}(t-s) \cdot x_{1}(s) d s .
$$

Substituting the expression (3) into the formula (4), we conclude that

$$
y(t)=\int m_{2}(t-s) \cdot m_{1}(s-u) \cdot x_{0}(u) d s d u,
$$

i.e., that

$$
y(t)=\int m(t-u) \cdot x_{0}(u) d u,
$$

where we denoted

$$
m(t)=\int m_{1}(s) \cdot m_{2}(t-s) d s .
$$

The formula (6) is known as the convolution of two functions $m_{1}(t)$ and $m_{2}(s)$ corresponding to the two layers.

Similarly, the joint effect of several layers can be described by a formula (5) in which the corresponding function $m(t)$ is a convolution of functions $m_{i}(t)$ corresponding to all the layers. So, to describe the joint effect of propagating the signal through several layers, we need to describe convolutions of several functions.

How to describe convolutions of several functions? Let us use the fact that a similar problem has been solved in probability theory. To describe the convolution of several functions, we can use the fact that a similar problem has already been solved in another area of applied mathematics - namely, in probability theory; see, e.g., [17].

Namely, it is known that if we have two independent random variables $x_{1}$ and $x_{2}$ described by the probability density functions (pdf's) $\rho_{1}\left(x_{1}\right)$ and $\rho_{2}\left(x_{2}\right)$, then the probability density function $\rho(x)$ describing their sum $x=x_{1}+x_{2}$ is equal to the convolution of the probability density functions corresponding to these variables:

$$
\rho(x)=\int \rho_{1}\left(x_{1}\right) \cdot \rho_{2}\left(x-x_{1}\right) d x_{1} .
$$

Similarly, the probability distribution function $\rho(x)$ corresponding to the sum $x=x_{1}+\ldots+x_{N}$ of a large number $N$ of independent random variables described by pdf's $\rho_{i}\left(x_{i}\right)$ is equal to the convolution of all $n$ pdf's $\rho_{i}\left(x_{i}\right)$.

So, from the mathematical viewpoint, analyzing the effect of propagating a signal through many layers is equivalent to analyzing the distribution of the sum of several random variables. For the sum of random variables, the distribution is known: namely, according to the Central Limit Theorem, if we have a large number of small independent random variables, then the distribution for their sum is close to Gaussian (normal) - and the more variables we add, the closer it it to normal.

The zone through which a seismic signal propagates can be divided into as many layers as we want - by simply considering narrower and narrower layers. When the layer is very thin, it practically does not affect the signal, i.e., its effect $m_{i}(t)$ is located in the close vicinity of 0 . In probabilistic terms, if the non-zero values of a pdf $\rho_{i}\left(x_{i}\right)$ are located mostly in the vicinity of $x_{i} \approx 0$, this means that the corresponding random variable is indeed small. Thus, the desired convolution is equivalent to the sum of large number independent small 
random variables - and hence, the joint effect is indeed described by the Gaussian formula

$$
m(t)=C \cdot \exp \left(-\frac{t^{2}}{2 \sigma^{2}}\right)
$$

for appropriate values $C$ and $\sigma$; see, e.g., [15].

Fourier transform helps to compute convolution. According to the formula (5), the observed signal $y(t)$ is the convolution of the original signal $x(t)$ and the functions $m(t)$ describing the layers through which the signal propagates.

From the computational viewpoint, computing the convolution directly is rather time-consuming: if we know the values $x(t)$ and $m(t)$ at $n$ moments of time, then, to compute each of $n$ values $y(t)$ corresponding to these moments of time, we need to perform $n$ multiplications $m(t-s) \cdot x(s)$ and $n$ additions adding these products together. Since we need $n$ computational steps for each of $n$ values $y(t)$, we thus need $n \cdot n=n^{2}$ computational steps to compute all the values $y(t)$, i.e., to compute the entire transformed signal. For large $n$, this may take too long.

We can, however, speed up computations by taking into account that the Fourier transform of the convolution is simply equal to the product of Fourier transforms. This fact is the easiest to illustrate on the example of convolution of pdfs. A Fourier transform of a function $f(x)$ is defined as the integral

$$
F(\omega)=\int \exp (-\mathrm{i} \cdot \omega \cdot x) \cdot f(x) d x
$$

where we denoted $\mathrm{i} \stackrel{\text { def }}{=} \sqrt{-1}$. In particular, when $f(x)$ is the probability density function $\rho(x)$, we get

$$
F(\omega)=\int \exp (-\mathrm{i} \cdot \omega \cdot x) \cdot \rho(x) d x .
$$

One can see that this integral is equal to the expected value of the corresponding exponential function:

$$
F(\omega)=E[\exp (-\mathrm{i} \cdot \omega \cdot x)] .
$$

Similarly, the Fourier transforms $F_{1}(\omega)$ and $F_{2}(\omega)$ of the probability density functions $\rho_{1}(x)$ and $\rho_{2}(x)$ can be described as

$$
\begin{gathered}
F_{1}(\omega)=E\left[\exp \left(-\mathrm{i} \cdot \omega \cdot x_{1}\right)\right] \text { and } \\
F_{2}(\omega)=E\left[\exp \left(-\mathrm{i} \cdot \omega \cdot x_{2}\right)\right] .
\end{gathered}
$$

When $x=x_{1}+x_{2}$, then

$$
\exp (-\mathrm{i} \cdot \omega \cdot x)=\exp \left(-\mathrm{i} \cdot \omega \cdot x_{1}\right) \cdot \exp \left(-\mathrm{i} \cdot \omega \cdot x_{2}\right) .
$$

Since $x_{1}$ and $x_{2}$ are independent, the expected value of the product is equal to the product of expected values:

$E[\exp (-\mathrm{i} \cdot \omega \cdot x)]=E\left[\exp \left(-\mathrm{i} \cdot \omega \cdot x_{1}\right)\right] \cdot E\left[\exp \left(-\mathrm{i} \cdot \omega \cdot x_{2}\right)\right]$,

i.e., indeed, $F(\omega)=F_{1}(\omega) \cdot F_{2}(\omega)$.

Thus, in terms of the Fourier transforms $\hat{x}(\omega)$ of the original signal $x(t)$ and $\hat{y}(\omega)$ of the propagated signal $y(t)$, the relation (5) takes the form $\hat{y}(\omega)=\hat{m}(\omega) \cdot \hat{x}(\omega)$, where $\hat{m}(\omega)$ is the Fourier transform of the Gaussian function (7). It is known that the Fourier transform of the Gaussian function (7) is also Gaussian,

$$
\hat{m}(\omega)=\text { const } \cdot \exp \left(-\frac{1}{2} \cdot \sigma^{2} \cdot \omega^{2}\right) .
$$

Thus, we conclude that

$$
\hat{y}(\omega)=\text { const } \cdot \exp \left(-\frac{1}{2} \cdot \sigma^{2} \cdot \omega^{2}\right) \cdot \hat{x}(\omega) \text {. }
$$

Relation between power spectra. We are interested in the power spectrum, which is the square of the absolute value of Fourier transform. The power spectra for the original and the propagated signals are, by definition, equal to $X(\omega) \stackrel{\text { def }}{=}$ $|\hat{x}(\omega)|^{2}$ and $Y(\omega) \stackrel{\text { def }}{=}|\hat{y}(\omega)|^{2}$. By taking the absolute value of both sides of the formula (8) and squaring the results, we conclude that the power spectrum $X(\omega)$ of the original signal and the power spectrum $Y(\omega)$ of the propagated signal are connected by a formula

$$
Y(\omega)=\text { const } \cdot \exp \left(-\alpha \cdot \omega^{2}\right) \cdot X(\omega),
$$

where we denoted $\alpha \stackrel{\text { def }}{=} \sigma^{2}$.

Let us list reasonable requirement for an approximating function. We are now ready to formulate the requirements on the approximating function.

We want to select a function $F(\omega)$ that describes observed power spectrum of the seismic signal $x(t)$.

The approximating function should be non-negative. First, by definition, power spectrum $X(\omega)$ is always non-negative: $X(\omega) \geq 0$.

It is therefore reasonable to require that the approximating function should also be non-negative: $F(\omega) \geq 0$.

The approximating functions should be differentiable. A single seismic signal comes from a single event - be it an earthquake or an artificially set explosion. This signal quickly fades with time. It is known that when a signal $x(t)$ is limited in time, its Fourier transform $\hat{x}(\omega)$ is differentiable infinite many times. Thus, its power spectrum $X(\omega)=\hat{x}(\omega) \cdot(\hat{x}(\omega))^{*}$, where $z^{*}$ means complex conjugation, is also differentiable.

It is therefore reasonable to require that the approximating function $F(\omega)$ be differentiable infinitely many times.

We should select a family of approximating functions. A seismic signal can have different amplitude. So, if $x(t)$ is a reasonable seismic signal, then $C \cdot x(t)$ is also a reasonable seismic signal, for any constant $C$.

For the signal $x(t)$, the power spectrum is equal to $X(\omega)=$ $|\hat{x}(\omega)|^{2}$. When we replace the signal $x(t)$ with the new signal $x^{\prime}(t)=C \cdot x(t)$, the Fourier transform is multiplied by the same constant $C$ and thus, the power spectrum is multiplied by $C^{2}$. So, if $X(\omega)$ is a reasonable power spectrum, then $C^{2} \cdot X(\omega)$ is also a reasonable power spectrum. Any positive real number can be represented as $C^{2}$; thus, we can describe this property in a simpler way: if $X(\omega)$ is a reasonable power spectrum, and $K>0$, then $K \cdot X(\omega)$ is also a reasonable power spectrum. 
If a function $F(\omega)$ is a good approximation to the power spectrum $X(\omega)$, then for the spectrum $K \cdot X(\omega)$, it is reasonable to use an approximation $K \cdot F(\omega)$. In these terms, what we want to select is not a single approximating function $F(\omega)$, but rather a family of functions $\{K \cdot F(\omega)\}_{K}$ corresponding to different values $K>0$.

The approximating family should allow different time scales. Similarly, some seismic events are happening faster, some are happening slower. If we simply "slow down" or "speed up" a reasonable seismic signal, by replacing $t$ with $t / c$ for some $c>0$, then we should also get a reasonable seismic signal. In other words, if $x(t)$ is a reasonable seismic signal, then $x(t / c)$ is also a reasonable seismic signal, for any constant $c>0$.

For the signal $x(t / c)$, its Fourier transforms is equal to const $X(c \cdot \omega)$. Thus, if $X(\omega)$ is a reasonable power spectrum, and $c>0$, then const $X(c \cdot \omega)$ is also a reasonable power spectrum. We already know that multiplication also leads to a reasonable power spectrum, so we conclude that $X(c \cdot \omega)$ is also a reasonable power spectrum.

If a function $F(\omega)$ is a good approximation to the power spectrum $X(\omega)$, then for the spectrum $X(c \cdot \omega)$, it is reasonable to use an approximation $F(c \cdot \omega)$. Because of this, what we really want to select is a family of functions

$$
\{K \cdot F(c \cdot \omega)\}_{K, c}
$$

corresponding to different values $K>0$ and $c>0$.

Propagation of the seismic signal should keep us within the approximating family. We want to approximate observed energy spectra. Different observed energy spectra $Y_{i}(\omega)$ are obtained from the energy spectrum $X(\omega)$ by the formula (9) corresponding to different values $\alpha$ :

$$
\begin{aligned}
& Y_{1}(\omega)=\text { const } \cdot \exp \left(-\alpha_{1} \cdot \omega^{2}\right) \cdot X(\omega) ; \\
& Y_{2}(\omega)=\text { const } \cdot \exp \left(-\alpha_{2} \cdot \omega^{2}\right) \cdot X(\omega) .
\end{aligned}
$$

Without losing generality, we can assume that $\alpha_{1}<\alpha_{2}$. In this case, from formulas (10a) and (10b), we conclude that

$$
Y_{2}(\omega)=\exp \left(-\alpha \cdot \omega^{2}\right) \cdot Y_{1}(\omega)
$$

where $\alpha \stackrel{\text { def }}{=} \alpha_{2}-\alpha_{1}$.

So, if $X(\omega)$ is a reasonable power spectrum, then the function

$$
\exp \left(-\alpha \cdot \omega^{2}\right) \cdot X(\omega)
$$

(corresponding to additional propagation) is also a reasonable power spectrum of the seismic signal.

It is therefore reasonable to require that if $F(\omega)$ is an approximating function, then, for every $\alpha>0$, this approximation $\exp \left(-\alpha \cdot \omega^{2}\right) \cdot F(\omega)$ should also belong to the approximating family $\{K \cdot F(c \cdot \omega)\}_{K, c}$, i.e., that for every $\alpha$, there should be values $K(\alpha)$ and $c(\alpha)$ (depending on $\alpha$ ) for which

$$
\exp \left(-\alpha \cdot \omega^{2}\right) \cdot F(\omega)=K(\alpha) \cdot F(c(\alpha) \cdot \omega) .
$$

Since the function $F(\omega)$ is differentiable, it is reasonable to require that the functions $K(\alpha)$ and $c(\alpha)$ should also be differentiable.
For $\alpha=0$, there is no need for changing anything, so we should have $K(0)=1$ and $c(0)=1$.

We are now ready to formulate our problem in precise terms.

\section{DEFinitions AND ThE MAIN RESUlt}

Definition 1. By an approximating family, we mean a family of functions

$$
\{K \cdot F(c \cdot \omega)\}_{K, c},
$$

where $F(\omega) \geq 0$ is a non-negative function which is differentiable infinitely many times, and $K$ and c can be any positive numbers.

\section{Comment.}

- The condition that $F(\omega) \geq 0$ comes from the fact that we want to approximate power spectra, and power spectrum is, by definition, non-negative.

- The condition that the function $F(\omega)$ be differentiable comes from the fact that we are approximating a wavelet, i.e., a signal which is bounded in time.

- The requirement that the approximating family contain functions $K \cdot F(\omega)$ comes from the fact that seismic signals can have different amplitude.

- The requirement that the approximating family contain functions $F(c \cdot \omega)$ comes from the fact that seismic signals can describe slower or faster processes.

\section{Definition 2. We say that an approximating family}

$$
\{K \cdot F(c \cdot \omega)\}_{K, c}
$$

is propagation invariant if for every $\alpha>0$, the equality (12) is satisfied for some differentiable functions $K(\alpha)$ and $c(\alpha)$ for which $K(0)=c(0)=1$.

Comment. This requirement means that if the approximating family is good for approximating signals coming after the first propagation stage, then this same family should also adequately describe signals which emerge from further propagation stages.

Discussion. One can check that the family corresponding to the Ricker wavelet function $F(\omega)=\omega^{2} \cdot \exp \left(-\omega^{2}\right)$ is propagation invariant: namely,

$$
\begin{gathered}
\exp \left(-\alpha \cdot \omega^{2}\right) \cdot F(\omega)=\omega^{2} \cdot \exp \left(-(1+\alpha) \cdot \omega^{2}\right)= \\
\frac{1}{1+\alpha} \cdot\left((1+\alpha) \cdot \omega^{2} \cdot \exp \left(-(1+\alpha) \cdot \omega^{2}\right)\right)= \\
K(\alpha) \cdot F(c(\alpha) \cdot \omega),
\end{gathered}
$$

for $K(\alpha)=\frac{1}{1+\alpha}$ and $c(\alpha)=\sqrt{1+\alpha}$. Let us now show that the Ricker wavelet is (almost) uniquely determined by the property of propagation invariance: namely, that it is one of the simplest approximating families with this property. 
Proposition. Every propagation-invariant approximating family corresponds to the function $F(\omega)=\omega^{2 n} \cdot \exp \left(-\omega^{2}\right)$ for some natural number $n=0,1, \ldots$

Discussion. The simplest case is $n=0$. In this case, we get the signals which correspond to a propagation of a simple pulse and thus, does not reflect the specifics of the original signal.

The simplest non-trivial case is $n=1$, which is exactly the Ricker wavelet.

Proof. We know that all the functions $F(\omega), K(\alpha)$, and $c(\alpha)$ are differentiable. Thus, we can differentiate both sides of the formula (12) with respect to $\alpha$. As a result, we get the following equality:

$$
\begin{gathered}
-F(\omega) \cdot \exp \left(-\alpha \cdot \omega^{2}\right) \cdot \omega^{2}= \\
K^{\prime}(\alpha) \cdot F(c(\alpha) \cdot \omega)+K(\alpha) \cdot F^{\prime}(c(\alpha) \cdot \omega) \cdot c^{\prime}(\alpha) \cdot \omega,
\end{gathered}
$$

where $K^{\prime}(\alpha)$ denotes the derivative.

In particular, for $\alpha=0$, we use the fact that $K(0)=$ $c(0)=1$ to get

$$
-F(\omega) \cdot \omega^{2}=k \cdot F(\omega)+F^{\prime}(\omega) \cdot c \cdot \omega,
$$

where we denoted $k \stackrel{\text { def }}{=} K^{\prime}(0)$ and $c \stackrel{\text { def }}{=} c^{\prime}(0)$. By moving all the terms proportional to $F(\omega)$ to the left-hand side, we get

$$
F \cdot\left(-k-\omega^{2}\right)=c \cdot \frac{d F}{d \omega} \cdot \omega .
$$

To solve this differential equation, let us separate the variables, i.e., by multiplying both sides by appropriate factors, let move all the terms $d F$ and $F$ to the right-hand side and all the other terms (including the terms related to $\omega$ and $d \omega$ ) to the left-hand side. Then, we get:

$$
\frac{1}{c} \cdot \frac{-k-\omega^{2}}{\omega}=\frac{d F}{F},
$$

i.e.,

$$
-\frac{k}{c} \cdot \frac{1}{\omega}-c \cdot \omega=\frac{d F}{F},
$$

Integrating both sides, we get

$$
C-\frac{k}{c} \cdot \ln (\omega)-\frac{c}{2} \cdot \omega^{2}=\ln (F),
$$

for some integration constant $C$. By exponentiating both sides, we conclude that

$$
F(\omega)=A \cdot \omega^{b} \cdot \exp \left(-B \cdot \sigma^{2}\right),
$$

where $A=\exp (C), b=-\frac{k}{c}$, and $B=\frac{c}{2}$.

The requirement that this function be differentiable for $\omega=0$ leads to the conclusion that $b$ is a natural number. The requirement that $F(\omega)$ is non-negative means that $b$ is an even natural number, i.e., that $b=2 n$ for some natural number $n$.

The proposition is proven.

\section{CONClusions And Future Work}

Conclusions. A natural way to process dynamic signals is to approximate these signals with functions from an appropriate family. In this paper, we consider the problem of processing seismic data. For this problem, we formulated reasonable requirements for approximating functions, and we showed that the simplest family of functions satisfying these requirements is the family of Ricker wavelets. This theoretical result is in good accordance with empirical findings - that in many cases, the use of Ricker wavelets enables us to use fewer parameters to approximate the same seismic signals with the given accuracy.

Future work. While in many cases, Ricker wavelet provide a very good approximation for seismic data, in some cases, the approximation quality of Ricker wavelets needs improvement. Such cases seem to indicate that it is not always sufficient to use the simplest possible approximate family of functions, that more complex approximating functions are sometimes needed. It is therefore desirable to find the best of such more complex approximating families - similar to how we found that the best of the simplest approximating families consists of Ricker wavelets.

\section{ACKNOWLEDGMENTS}

This work was supported in part by the National Science Foundation grants HRD-0734825 and HRD-1242122 (CyberShARE Center of Excellence) and DUE-0926721.

The authors are thankful to Laura Serpa for her support and encouragement, and to the anonymous referees for valuable suggestions.

\section{REFERENCES}

[1] M. Apostolou, G. Gazetas, and E. Garini, "Seismic response of slender rigid structures with foundation uplifting", Soil Dynamics and Earthquake Engineering, 2007, Vol. 27, pp. 642-654.

[2] A. E. Barnes, "Instantaneous spectral bandwidth and dominant frequency with applications to seismic reflection data", Geophysics, 1993, Vol. 58, No. 3, pp. 419-428.

[3] A. Chakraborty and D. Okaya, "Frequency-time decomposition of seismic data using wavelet-based methods", Geophysics, 1995, Vol. 60, No. 6, pp. 1906-1916.

[4] X. Deng, Y. Li, and B. Yang, "Ricker wavelet LS-SVM and its parameter setting for seimsic prospecting signals denoising”, In: C. Wang, S. Zhong, and J, Wei (eds.), Proceedings of the Second International Conference on Space information Technology, SPIE Proceedings, 2007, Vol. 6795, Paper $1 \mathrm{H}$.

[5] N. Economou, A. Vafidis, H. Hamdan, G. Kritikakis, N. Andronikidis, and K. Dimitriadis, "Time-varying deconvolution of GPR data in civil engineering", Nondestructive Testing and Evaluation, 2012, Vol. 27, No. 3, pp. 285-292.

[6] N. Gerolymos, M. Apostolou, and G. Gazetas, "Neural network analysis of overturning response under near-fault type excitation", Earthquake Engineering and Engineering Vibration, 2005, Vol. 4, No. 2, pp. 213228.

[7] O. Kosheleva, S. D. Cabrera, G. A. Gibson, and S. Cherukuri, "Interval estimates for signal processing: special purpose hardware", Reliable Computing, 1999, Vol. 5, No. 2, pp. 175-196.

[8] R. B. Latimer, R. Davison, and P. van Riel, “An interpreter's guide to understanding and working with seismic-derived acoustic impedance data", The Leading Edge, 2000, Vol. 19, No. 3, pp. 242-255. 
[9] J. Liu, Y. Wu, D. Han, and X. Li, "Time-frequency decomposition based on Ricker wavelets", Proceedings of the 74th Annual International Meeting of the Society of Exploratory Geophysicists SEG'2004, Denver, Colorado, October, 10-15, 2004, pp. 1937-1940.

[10] Y. Liu and S. Fomel, "Seismic data analysis using local time-frequency transform", Geophysical Prospecting, 2013, Vol. 61, pp. 516-525.

[11] M. Loli, I. Anastasopoulos, J. A. Knappett, and M. J. Brown, "Use of Ricker wavelet ground motions as an alternative to push-over testing", Proceedings of the 8th International Conference on Physical Modelling in Geotechnics ICPMG'2014, Perth, Australia, January 14-17, 2014, pp. 1073-1078.

[12] M. D. McCormack, D. E. Zaucha, and D. W. Dushek, "First-break refraction event picking and seimsic data trace editing using neural networks", Geophysics, 1993, Vol. 58, No. 1, pp. 67-78.

[13] J. M. Mendel, "White-noise estimators for seismic data processing in oil exploration", IEEE Transactions on Automatic Control, 1977, Vol. AC22, No. 5, pp. 694-706.
[14] W. Menke, Geophysical Data Analysis: Discrete Inverse Theory, Academic Press, Waltham, Massachusetts, 2012.

[15] O. Ochoa, M. Ceberio, and V. Kreinovich, "How to describe spatial resolution: an approach similar to the central limit theorem", Applied Mathematical Sciences, 2010, Vol. 4, No. 63, pp. 3153-3160.

[16] N. Ricker, "The form and laws of propagation of seismic wavelets", Geophysics, 1953, Vol. 18, pp. 10-40.

[17] D. J. Sheskin, Handbook of Parametric and Nonparametric Statistical Procedures, Chapman \& Hall/CRC, Boca Raton, Florida, 2011.

[18] R. H. Wolfe and C. N. Liu, "Intractive visualization of 3D seismic data: a violumetric method", IEEE Computer Graphics and Applications, 1988, Vol. 8, No. 4, pp. 24-30.

[19] V. Zania, P. N. Psarropoulos, Y. Karabatsos, and Y. Tsompanakis, "Inertial distress of waste landfills", Computers \& Structures, 2008, Vol. 86, No. 7, pp. 642-651.

[20] C. Zhang and T. J. Ulrych, "Estimation of quality factors from CMP records", Geophysics, 2002, Vol. 67, No. 5, pp. 1542-1547. 